\title{
CRÍTICA AL DERECHO DE AUTOR EN LA ERA DE LA INFORMATIZACIÓN DE LA PRODUCCIÓN
}

\author{
CRITICISM OF AUTHOR COPYRIGHTS IN THE DIGITAL \\ AGE OF THE INFORMATIZATION OF PRODUCTION
}

\section{Claudio Celis ${ }^{1}$}

\section{RESUMEN}

El objetivo principal del texto es interpretar el fenómeno del derecho de autor en nuestra era actual desde la crítica a la propiedad privada desplegada por Marx en los $\mathrm{Ma}$ nuscritos económico-filosóficos de 1844. En el estado actual de la información digital, la posibilidad de reproducción y distribución del material protegido por el derecho de autor es puesto en crisis, revelando el conflicto fundamental sobre el que se sostiene toda ilusión de propiedad. Por último, el texto indaga las posibilidades críticas de estas nuevas condiciones de producción desde la distinción entre policía y política establecida por Jacques Rancière.

Palabras clave: Derecho de autor, piratería, propiedad intelectual, Marx, manuscritos económico-filosóficos, Rancière, era digital.

\section{ABSTRACT}

The main aim of this text is to analyze the concept of copyright in our present society using the Marxist critique of private property located in his 1844 manuscripts. In the present state of digital information, reproduction and distribution capabilities throw into crisis the traditional concept of copyright, thus revealing the conflict upon which it is built. Finally, the text inquires about the reflexive possibilities of this new age appealing to Rancière's distinction between police and politics.

Keywords: Copyright, piracy, intellectual property, Marx, 1844 manuscripts, Rancière, digital age.

Recibido: 27.12.10. Aceptado: 06.07.11.

${ }^{1}$ Magíster en Teoría e Historia del Arte de la Universidad de Chile. Académico e investigador de la Facultad de Arquitectura, Arte y Diseño de la Universidad Diego Portales. Santiago, Chile. E-mail: claudio.celis@udp.cl 
Yo lo repito: el autor es dueño de su obra, o no hay persona en la sociedad que sea dueña de sus bienes.

ON LA expansión de las tecnologías digitales, el problema del derecho de autor se ha convertido en un importante punto de discusión. Estas discusiones, sin embargo, se han limitado a la superestructura jurídica, reflexionando acerca de los límites en los usos y abusos de la propiedad intelectual, pero no cuestionando el estatuto mismo de la propiedad privada. Y si bien estas discusiones surgen de una realidad concreta (la posibilidad técnica de violentar las restricciones impuestas a la distribución y circulación de productos protegidos por la ley de derecho intelectual), se reducen a una búsqueda de soluciones prácticas que permitan combinar la cada vez mayor disponibilidad tecnológica con la cada vez más exigente reproducción de la plusvalía en el consumo cultural. Creo necesario, y en contra de este tipo de reflexiones, poner la mirada en la condición misma de la propiedad intelectual en tanto propiedad privada.

El acceso cada vez mayor a la producción cultural posibilitado por la expansión tecnológica y el consecuente crecimiento exponencial de la piratería traen en sí el germen que pone en crisis la "naturalización" ideológica del derecho de autor como propiedad inalienable. Esta crisis nos permite reflexionar una vez más sobre las condiciones históricas de la propiedad privada, denunciando así la hegemonía con la que ésta se ha "naturalizado" en el ámbito jurídico y social.

Para Marx, la economía política clásica parte del hecho de la propiedad privada, pero no la explica. En las discusiones actuales acerca del derecho de autor ocurre lo mismo. Reflexionan acerca de su funcionamiento al interior de un mundo que no deja de minar sus bases y, sin embargo, insisten ciegamente en su supervivencia sin siquiera cuestionar su origen. Tal como propone Marx, el edificio de la economía política se sostiene por completo sobre los cimientos del derecho "natural" a la propiedad privada; de igual modo, el capitalismo actual, en su estado inmaterial o de la informatización de la producción, sería impensable sin los mecanismos que aseguran la reproducción de la propiedad intelectual. Las grandes corporaciones farmacológicas o de desarrollo de software comercial, por ejemplo, se levantan en su totalidad sobre la posibilidad de otorgar un determinado valor de cambio a una "abstracción"; corporaciones que simplemente se derrumbarían sin los mecanismo legales que aseguran su propiedad sobre dicha abstracción.

Para desarrollar la crítica a la propiedad privada como tal utilizaré los 
argumentos desplegados por Marx en los manuscritos "económico-filosóficos" del '44, los cuales desplazaré posteriormente al caso puntual del derecho de autor en la era de la informatización de la producción. Por último, recurriré a la reflexión de Rancière acerca del concepto de "emancipación" para complejizar la crítica de Marx a la propiedad privada y para indagar en las posibilidades de una crítica actual.

\section{LOS MANUSCRITOS DEL '44: CRÍTICA A LA PROPIEDAD PRIVADA}

La Economía política, dice Marx (1968), "arranca del hecho de la propiedad privada, pero no la explica" (p. 73); y cuando intenta hacerlo, debe recurrir a "imaginarios estados primitivos" - robinsonadas - para hallar allí un pseudo-argumento que valide su origen. Con ello no hace más que "desplazar el problema hacia un remoto pasado oscuro y nebuloso" (Marx, 1968, p. 74). $\mathrm{Al}$ contrario, Marx pretende explicar el fenómeno de la propiedad privada partiendo de un hecho económico actual: el conflicto entre el trabajador asalariado y el capitalista².

En cuanto trabajador asalariado, el trabajador se enfrenta al objeto producido por su trabajo como algo extraño a él, el producto se presenta como un poder independiente del productor: "el producto del trabajo es el trabajo que se ha plasmado, materializado en un objeto, es la objetivación del trabajo. Esta realización del trabajo (objetivación) como estado económico, se manifiesta como la privación de la realidad del obrero" (Marx, 1968, p. 75). Este es el primer aspecto del trabajo alienado: "el obrero se comporta hacia el producto de su trabajo como hacia un objeto ajeno" (Marx, 1968, p. 75).

Si el primer aspecto del trabajo alienado se da al nivel de la relación entre el trabajador y el producto de su trabajo, el segundo aspecto se da en "el acto mismo de la producción, en la misma actividad productiva" (Marx, 1968, p. 77). La enajenación frente al objeto producido no es sino la manifestación de la enajenación en la actividad productiva misma. Marx (1968) plantea que "si el producto de trabajo es la enajenación, la producción mis-

\footnotetext{
${ }^{2}$ Según Marx, la sociedad capitalista se levanta sobre la contradicción esencial, la "lucha antagónica" entre el capitalista y el obrero. El salario es la manifestación real de esta lucha entre quienes poseen los medios de producción y aquellos que sólo poseen su fuerza de trabajo. El salario es el precio del trabajo, pero en tanto este trabajo ha sido objetivado como mercancía. El capital, por su parte, no es más que trabajo acumulado, que ofrece además la capacidad de comprar más trabajo.
} 
ma tiene que ser necesariamente la enajenación activa, la enajenación de la actividad, la actividad de la enajenación" (p. 78). Marx se pregunta, entonces, ¿de dónde proviene la enajenación de la actividad productiva misma? En primer lugar, dice, del hecho de que el trabajo es concebido como algo externo al trabajador y no como algo que forma parte de su esencia. El segundo aspecto de la enajenación consiste en que la propia actividad productiva pasa de ser una esencia del hombre a ser un mero medio para su existencia. La importancia del análisis del trabajo enajenado, en tanto objetivación externa de su propia actividad, reside entonces en que permite comprender la esencia del hombre, su ser genérico.

El análisis del trabajo enajenado revela algo acerca de la esencia de la actividad vital del hombre: "la actividad vital consciente distingue al hombre directamente de la actividad vital de los animales. Y ello es precisamente lo que hace de él un ser genérico [...] es sólo y precisamente en la transformación del mundo objetivo donde el hombre comienza a manifestarse realmente como ser genérico" (Marx, 1968, p. 81). La actividad productiva humana es la externalización, objetivación, de su voluntad y conciencia. Por esta razón, "el trabajo enajenado invierte los términos de la relación, en cuanto que el hombre, precisamente porque es un ser consciente, hace de su actividad vital, de su esencia, simplemente un medio para su existencia" (Marx, 1968, p. 81). El trabajo enajenado convierte lo propio del ser genérico -su actividad vital- en un medio para su existencia individual, en un ser extraño a sí mismo³.

Es fundamental aclarar que la transformación del mundo por parte del hombre (su actividad vital) implica siempre su existencia en tanto sujeto social: el ser genérico es siempre un ser social inscrito históricamente. El problema del trabajo alienado es precisamente que se desarrolla al interior del antagonismo entre el trabajador y el capitalista, es decir, entre el obrero y el no-obrero. Esta oposición es el origen de la enajenación del trabajo y por ende de la propiedad privada, y no a la inversa como lo pretende la economía política. Si para esta última la propiedad privada es el origen de la economía burguesa, para Marx ésta es sólo la consecuencia de determinado

\footnotetext{
${ }^{3}$ Para Hegel, la alienación -la externalización de la conciencia en un objeto distinto de ella- es un momento necesario para el "progreso del espíritu": el hombre necesita "objetivar" su voluntad en un objeto diferente de sí para poder luego aprehenderla sensiblemente. Para Marx, en cambio, la actividad productiva no es de por sí alienante, sino sólo en determinadas condiciones productivas (división del trabajo). Esto marca un giro desde la concepción "antropológica” de la alienación (Hegel) hacia una interpretación "histórica" de ésta (Marx). Esta sería al menos la hipótesis de Ernest Mandel en su libro La formación del pensamiento económico de Marx.
} 
modo de producción, su origen es la alienación del trabajo: "La propiedad privada es, pues, el producto, el resultado, la consecuencia necesaria del trabajo enajenado, de la relación externa del obrero con la naturaleza y consigo mismo" (Marx, 1968, p. 85).

En los manuscritos, Marx parte de la crítica a la economía política para desentrañar de ella la esencia de la propiedad privada, del trabajo alienado y por último del hombre en tanto ser genérico. Esto lo hace a partir de la interpretación de un fenómeno social concreto -la lucha antagónica entre el trabajador y el capitalista- y no recurriendo a "robinsonada" alguna. Continuando con la crítica a la economía política, Marx propone que para ésta, en especial para Adam Smith, "la esencia subjetiva de la propiedad privada, la propiedad privada como actividad de por sí, como sujeto, como persona, es el trabajo" (Marx, 1968, p. 103). La novedad introducida por Smith reside en que ya no comprende la propiedad privada como algo exterior al hombre, sino como la actividad humana hecha objeto ${ }^{4}$.

Es importante remarcar que este proceso de internalización de la esencia de la propiedad privada es una consecuencia del desarrollo de la industria moderna y no una invención del "genio de Adam Smith". Si consideramos que sólo en el modo de producción industrial puede el hombre liberarse de toda riqueza externa -la tierra por ejemplo-, y reducir el origen de la propiedad privada al trabajo, y si bien éste es el verdadero "golpe mortal" de la economía política, ya que naturaliza la negación total del hombre -"la esencia de la propiedad privada hecha conflicto"-, debemos insistir en que sólo a través de esta propia enajenación provocada por la industria moderna puede el hombre tomar conciencia de su esencia en tanto ser genérico y con ello reapropiarse de su propia actividad productiva. De este modo, el grado de desarrollo de la contradicción entre el capital y el trabajador alcanza un estado tal en la industria moderna y en la moderna Economía Política que impulsa ella misma a su solución: la superación positiva ("aufhebung") de la propiedad privada sólo es posible porque su esencia ha sido objetivada, externalizada, en la industria moderna. A la hora de pensar esta superación, Marx propone dos tipos de comunismo. El "comunismo vulgar" (tosco, grosero) y el "comunismo real". El primero de ellos refiere a un tipo de sociedad en el cual la esencia de la propiedad privada no ha sido efec-

\footnotetext{
${ }^{4}$ Marx ejemplifica este fenómeno recurriendo a Lutero: tal como él ha internalizado y subjetivado la religión, Smith acaba con la riqueza como algo externo al hombre y funda su origen en su propia esencia: "Así como Lutero negó al cura fuera del lego, transponiéndolo al corazón de éste, la Economía Política acaba con la riqueza situada fuera del hombre e independiente de él, para incorporar la propiedad privada al hombre mismo" (Marx, 1968, p. 104).
} 
tivamente superada, sino que el Estado ejerce una tarea distributiva. Todo lo apropiable es apropiado por la comunidad, la cual funciona como "una comunidad de trabajo" en la cual existe una igualdad de salario asegurada por esta misma comunidad en tanto "capitalista general". El "comunismo real", en cambio, es una "superación positiva" -en sentido hegeliano- de la propiedad privada. En tanto tal sólo es posible en la medida en que se ha captado la esencia del hombre como ser genérico (transformación del mundo mediante la actividad humana, al interior del ser social y como parte de un desarrollo histórico). El comunismo real es la apropiación de la esencia humana por y para el hombre: superación de la autoenajenación: "verdadera solución del conflicto entre el hombre y la naturaleza y del hombre contra el hombre, la verdadera solución de la pugna entre la existencia y la esencia, entre la objetivación y la afirmación de sí mismo, entre la libertad y la necesidad, entre el individuo y la especie" (Marx, 1968, p. 114).

El objetivo de Marx es refutar la teoría de la economía política respecto de la propiedad privada. En el Segundo Tratado sobre el Gobierno Civil ([1689] 1986), John Locke argumenta que si bien en un origen no existe la propiedad privada en tanto tal, existe desde siempre un bien que es inalienable a cada individuo: su propio cuerpo y la fuerza de éste. De esta propiedad primera se deriva que cada cosa que ese cuerpo extraiga de la naturaleza -o que produzca a partir de ésta- pasará a ser de su posesión. La Economía política burguesa utilizará este argumento para justificar el derecho "natural" a la propiedad privada.

En contra de esta argumentación los Manuscritos nos permiten plantear: a) que este argumento se funda sobre una fantasía originaria, un "imaginario estado primitivo", en vez de partir del análisis real de la sociedad actual; b) que la propiedad privada, más que un derecho inalienable y ahistórico, es el producto concreto de determinado modo de producción; y c) que la propia apelación a la propiedad del cuerpo como origen de la propiedad privada debe ser puesta en duda a partir de la definición del hombre en tanto ser genérico. Este último punto resulta fundamental para una crítica a la propiedad privada en general, y a la propiedad intelectual en particular.

Mientras para Locke el cuerpo constituye ese límite de la individualidad

\footnotetext{
${ }^{5}$ Para ejemplificar el comunismo vulgar, Marx recurre al caso de la mujer: si el matrimonio representa a la mujer en tanto propiedad privada, entonces el comunismo vulgar dispondría de la mujer como "prostituta universal". En este ejemplo, la relación de "apropiación" no varía entre el matrimonio (economía burguesa) y la prostitución (comunismo vulgar), sólo se distribuye equitativamente el objeto apropiado (Marx, 1968, p. 112).
} 
que asegura la apropiación del mundo, para Marx nuestro cuerpo es siempre el producto del contexto histórico y social en el cual ha sido conformado. El ejemplo más evidente aquí son los sentidos: "los sentidos del hombre social son otros que los del hombre no social" (Marx, 1968, p. 121). Si consideramos que toda producción de objeto es también la producción de un sujeto para ese objeto (ya que todo objeto va modificando nuestros sentidos) y si consideramos además que toda producción de objeto es siempre la producción mía para otro o de otro para mí, entonces debemos decir que el desarrollo de los "sentidos humanos" del ser genérico se modifica en la medida en que se desarrolla la totalidad social. Los diferentes modos de producción producen diferentes modos de sensibilidad. Por ello que cada vez que gozamos en tanto humanos debemos dicho goce al desarrollo de nuestros sentidos subjetivos: "el oído musical, el ojo capaz de captar la belleza de la forma" son formaciones históricas posibilitadas por el desarrollo de las fuerzas productivas que son a su vez el ser genérico del hombre objetivado. Por ello que "la formación de los cinco sentidos sea la obra de toda la historia universal anterior" (Marx, 1968, p. 121).

La música es uno de los ejemplos más palpables de este argumento: para gozar de una pieza musical, mi oído debe haber sido disciplinado y culturizado, lo cual sólo ocurre como fenómeno inscrito social e históricamente. De igual modo, a la hora de producir una pieza musical, el compositor deberá su "genio" a la mano que ha aprendido a manejar un instrumento y al oído que ha aprendido a guiarla. El ejemplo del goce artístico como goce social impide apelar a cualquier apropiación de la creación intelectual como propiedad privada, ya que refuta desde la base cualquier individualidad originaria.

Con este argumento, los Manuscritos refutarían la teoría de Locke respecto del cuerpo como origen de la propiedad privada. Los sentidos del hombre, en tanto "sentidos humanos", son ya el producto de mi relación con la realidad social, la cual a su vez es el producto de la actividad productiva de los otros. Cuando utilizo dichos sentidos tanto para producir objetos como para gozarlos, la totalidad del ser social se encuentra comprometida en ello. Sin embargo, "es necesaria la objetivación de la esencia humana, tanto en el aspecto teórico como en el práctico, lo mismo para convertir en humano el sentido del hombre como para crear el sentido humano adecuado a toda la riqueza de la esencia humana y natural" (Marx, 1968, p. 121). Esta objetivación tanto teórica como práctica sólo puede manifestarse en el campo de la economía, más precisamente en la industria moderna.

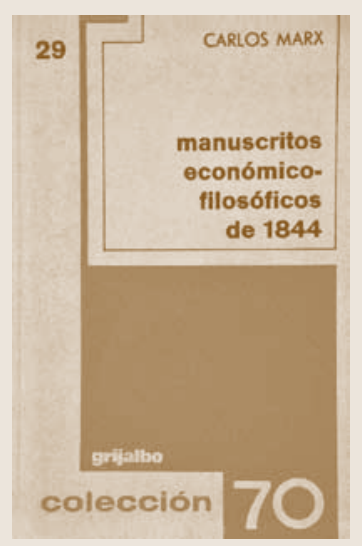


Insistamos: sólo el desarrollo de la industria ha permitido al hombre aprehender sensiblemente su ser genérico objetivado: "la historia de la industria y la existencia objetiva de la industria, ya hecha realidad, es el libro abierto de las fuerzas esenciales humanas, la psicología humana colocada ante nuestros sentidos, que hasta ahora no se concebía como entroncada con la esencia del hombre, sino siempre en un plano externo de utilidad [...] En la industria usual, material, tenemos ante nosotros, bajo la forma de objetos útiles sensibles y ajenos, bajo la forma de la enajenación, las fuerzas esenciales objetivadas del hombre" (Marx, 1968, p. 122). Y si bien la industria moderna ha posibilitado el estado de máxima enajenación del trabajo (el trabajo como fundamento subjetivo de la propiedad privada), es también la industria, como esencia humana objetivada, la que permitirá la superación positiva de la propiedad privada.

\section{DEL DERECHO DE AUTOR, LA PIRATERÍA Y LAS NUEVAS TECNOLOGÍAS}

El análisis de la propiedad privada, del ser genérico y de la industria moderna desplegado en los Manuscritos nos permite dar nuevas luces al problema de la propiedad intelectual en la era de su producción, distribución y consumo digital. A grandes rasgos, el problema es el siguiente: el acceso cada vez mayor a las tecnologías digitales permite un acceso cada vez mayor a los productos culturales; esta doble accesibilidad pone en crisis el estatuto mismo del derecho de autor, propicia una superación del binomio espectáculo- espectador, y permite concebir el trabajo creativo más allá de los límites de la propiedad privada, haciendo aparecer su condición de actividad genérica, social e histórica.

\section{A. Piratería y propiedad privada}

Dicho en términos generales, la piratería es la reproducción de material protegido por el derecho intelectual sin el consentimiento por parte del autor. Esta reproducción puede ser utilizada con fines comerciales como con fines "privados". La piratería en cuanto tal ha existido cada vez que los medios de reproducción técnica se tornan accesibles. La piratería de libros, por ejemplo, comienza ya en el siglo XVII, con la masificación de la imprenta. La piratería de música y de películas se masifica con la llegada al mercado 
de grabadores de CD y DVD respectivamente. La implementación del derecho de propiedad intelectual, por su parte, surge siempre como respuesta a esta masificación de los mecanismos de reproducción. Como propone $\mathrm{Wu}$ Ming, "las mismas corporaciones que venden samplers, fotocopiadoras, escáneres y quemadores de CD controlan la industria global del entretenimiento, que un día se descubre dañada por el uso de tales instrumentos. La serpiente se muerde la cola y entonces presiona a los diputados para que legislen en contra de la autofagia" (Wu Ming, 2005).

El derecho de propiedad sobre una producción intelectual no antecede a la piratería, sino que surge como respuesta a ella. Y así como Marx demostró que la propiedad privada no es el origen de la economía política, sino que surge de un modo de producción específico, de igual modo podemos plantear hoy que la propiedad intelectual no es el origen del consumo cultural, sino simplemente la manifestación, en determinada forma de producción, del conflicto entre el trabajo y el capital.

En términos económicos, la piratería, como infracción del copyright, interrumpe el momento de realización de la plusvalía del consumo cultural. El capitalista -léase: sello discográfico, editorial, estudio cinematográfico, productora, etc.- se apropia del trabajo de técnicos, artistas y profesionales para extraer de él su plusvalía. Ahora bien, para que dicha plusvalía se realice como ganancia, el capitalista debe llevar al mercado y vender las obras producidas. Este segundo momento de la reproducción del capital (necesario para completar el trinomio capital-mercancía-capital aumentado) es interrumpido por la piratería al permitir el consumo de la mercancía en cuestión sin pagar por ella -o pagando sólo por el costo de su reproducción-. Ante esta aberración económica, la superestructura jurídica responde con la protección del derecho de propiedad intelectual, heroica tarea que más que proteger al "artista" oculta la mundana necesidad de asegurar la reproducción del antagonismo entre capital y trabajo ${ }^{6}$.

Leída de este modo, la piratería se parece más a lo que Marx llamó el "comunismo vulgar" que a lo que él mismo pensó como "comunismo real". La piratería no apela a una superación real de la propiedad intelectual, sino a su mera libre distribución: no ha superado la concepción de los productos culturales como algo externo al trabajo, y por ende sigue considerándolos como objetos "apropiables". Su función efectiva es la de expandir la acce-

\footnotetext{
${ }^{6}$ En este sentido, la figura del autor y del derecho intelectual son dos figuras legales estrictamente modernas que nacen con el desarrollo del capitalismo como medio para poner precio al trabajo inmaterial.
} 
sibilidad a las mercancías de la industria cultural y no la superación del trabajo en tanto medio de subsistencia. En la piratería no hay destrucción y superación -"aufhebung"- de la propiedad privada, sólo hay redistribución de la propiedad en tanto algo externo, alienado, y por ende apropiable.

A pesar de esta constatación, podemos plantear que la piratería juega un papel fundamental, ya que si bien no modifica la relación con el objeto, hace aparecer el problema del derecho de autor y de la propiedad intelectual. Con ello, llama a reflexionar acerca del estatuto mismo de la propiedad privada en la era de la informatización de la producción. Y aun cuando la piratería no vaya más allá de una mera analogía con el "comunismo vulgar", su expansión digital representa el punto de partida para un posible giro en la concepción misma de la propiedad intelectual.

\section{B. La técnica digital como herramienta de emancipación}

El desarrollo de la industria en su fase digital -que coincide con el capitalismo tardío o de la informatización de la producción- es el grado más sofisticado en lo que a objetivación del trabajo humano respecta. La tecnología digital es el punto máximo de enajenación del trabajo, en la medida en que éste se percibe como un medio externo al hombre, y aquélla como un instrumento para su explotación. El capitalismo actual, con su alto grado de sofisticación y racionalización, sólo es posible gracias a los aportes de las herramientas digitales. La técnica informática -en tanto trabajo humano enajenado- posee una doble faceta: por un lado se convierte en un nuevo fetiche (fetichismo de la técnica, reproducción de pseudo-necesidades) que permite ampliar el territorio de expansión y reproducción del capital; por otro lado, se convierte en una herramienta fundamental de control social (estadísticas, proyecciones, vigilancia, etc.) que asegura la contradicción entre el capital y el trabajador. La industria moderna, en su fase actual, digital, es el máximo triunfo de la ideología capitalista: facilita el control de la producción y colabora con la reproducción del orden de la propiedad. Sin embargo, es esta misma objetivación la que permite -o puede permitir-su reapropiación, es decir, la superación positiva, real, de la propiedad privada. Marx llamaba a la industria "el libro abierto de las fuerzas humanas". Sólo cuando leamos la tecnología digital como la objetivación de la enajenación, como la separación entre el productor y su producto hecha objeto frente a nosotros, sólo allí tomaremos conciencia del rol que la tecnología juega en el proceso de superación de la propiedad privada. 
Con el desarrollo de los medios de almacenamiento y reproducción digital, sumado a la expansión radical de Internet, la totalidad de los objetos culturales pasa a conformar una gran base de datos. Con la digitalización del patrimonio cultural -en sentido lato- se está alcanzando un momento en la historia de la humanidad en el cual la totalidad de sus producciones "culturales" se encontrarán accesibles a cualquier usuario con un computador y una conexión a Internet. Y si bien la superestructura jurídica intenta restringir este acceso -le parece inconcebible la idea de un objeto no traducible en términos de valor de cambio-, la técnica misma rebasa toda posibilidad de limitación o control. En esta disponibilidad total nos vemos nuevamente enfrentados con la contradicción central del capitalismo: por un lado, el capital dispone de la totalidad de los productos culturales para realizar a través de ellos su reproducción; sin embargo, es esta misma hegemonía de los productos culturales la que hace aparecer, de modo objetivado y ante nuestros propios ojos, que toda producción es siempre producción social e histórica.

$\mathrm{Al}$ analizar la propiedad privada, Marx concluye que ésta no es el origen de la economía burguesa, sino el producto de un determinado tipo de producción: la propiedad privada no es un derecho inalienable del hombre, sino el producto de su trabajo alienado. En contra del argumento liberal de que el individuo es dueño, en última instancia de su cuerpo y de la actividad de dicho cuerpo, Marx argumenta que nuestros sentidos, en tanto sentidos humanos, son condicionados y modificados por el ser social. La tecnología digital, al poner ante nosotros el proceso de objetivación del trabajo humano, nos permite comprender el trabajo en su condición de actividad vital del ser genérico, y no como un simple medio para la subsistencia.

El desplazamiento del análisis expuesto en los Manuscritos desde la industria moderna decimonónica hacia el estado actual de la esfera productiva nos permite tomar la siguiente posición respecto de los productos del trabajo intelectual: a) que éstos no le pueden pertenecer a ningún individuo privado, ya que su producción es producto de toda la historia de la humanidad; b) mientras insistamos en los productos intelectuales como propiedad privada sólo reproduciremos la alienación inherente a la economía política que se relaciona con el trabajo como medio externo cuya única finalidad es asegurar la supervivencia; c) si, por el contrario, concebimos al hombre como ser genérico, y su trabajo como actividad vital, entonces es la producción misma de los productos intelectuales (en tanto objetos de goce inscritos siempre social e históricamente) lo que los justifica -y no su traducción en salario o en ganancia-. 
Llamaré prácticas de emancipación digital a las prácticas que no utilizan la piratería como mecanismo de ganancia ni de simple distribución, sino como una herramienta para realizar un trabajo inmaterial bajo una nueva lógica que supera la noción misma de propiedad privada. Ejemplos de estas prácticas son: a) el software libre de fuente abierta (GNU), proyecto que concibe el desarrollo de un software como tarea comunitaria y que para ello asegura no sólo su distribución libre, sino la distribución del código fuente de programación, permitiendo que cualquiera lo modifique y vuelva a compartir; b) las prácticas artísticas de postproducción que se apropian de material de archivo (protegido por el derecho de autor) para manipularlo y producir un nuevo significado; c) el nuevo estado de producción, distribución y consumo musical que permite prescindir del sello discográfico para grabar y compartir una obra.

Estos tres ejemplos representan tres prácticas en las cuales la relación con el trabajo y con los productos del trabajo ya no se concibe como una relación externa, de supervivencia, traducible a un salario o una ganancia. A diferencia de la piratería, que simplemente distribuye la propiedad privada, en este segundo momento, la noción misma de propiedad privada exige ser superada.

El optimismo del análisis marxista de la sociedad capitalista reside en proponer que la contradicción central que la sostiene, aquélla entre el desarrollo de las fuerzas productivas y su control, producirá ella misma las condiciones para su superación. Así, mientras la tecnología digital alimenta una industria cultural que nos mantiene como espectadores pasivos consumiendo su infinita cadena de mercancías, es el propio progreso técnico el que hace cada vez más accesible los medios de producción digitales, trayendo consigo la promesa de que los espectadores -usuarios pasivosdevengan productores -usuarios activos- que conciban su propio trabajo inmaterial más allá de la lógica de la economía política ${ }^{7}$.

El potencial crítico de las prácticas de emancipación digital consiste en hacer que los usuarios de estas nuevas tecnologías participen directamente

\footnotetext{
${ }^{7}$ Siendo rigurosos con el propio análisis marxista, debemos reconocer que esta etapa de superación de la propiedad privada en el ámbito del trabajo inmaterial es sólo un escalón en la superación real de la propiedad privada en la totalidad social. En la concepción "emancipada" del trabajo inmaterial, sigue existiendo una base productiva que asegura las condiciones de existencia de los individuos. Es el propio Marx, en algunos fragmentos de los manuscritos del '57-'59 (Grundrisse) que introduce la noción de "General Intellect” para pensar este problema: el intelecto general objetivado en la fábrica es el producto del trabajo alienado, pero es también lo que permitirá asegurar en el comunismo real las condiciones materiales de existencia de la totalidad social. (Véase: Marx, 2009).
} 
de los procesos de producción y de consumo y que aceleren con ello la disolución del binomio autor-espectador, binomio jurídico que asegura la explotación capitalista de los productos intelectuales y que mantiene anestesiado el rendimiento inherentemente revolucionario del progreso técnico.

\section{RANCIÈRE Y LOS LÍMITES DE LA EMANCIPACIÓN}

En Sobre la cuestión judía, Marx distingue entre la emancipación política y la emancipación humana. La primera, propone, es la emancipación de la sociedad civil respecto del poder señorial, y que instaura un régimen de libertad individual, egoísta, y una concepción de los derechos civiles del hombre como derechos naturales. Si bien Marx reconoce que la emancipación política es un gran paso histórico, su crítica se concentra en la despolitización que esta emancipación representa: el individuo privado de la sociedad civil, con sus derechos naturales y su libertad egoísta, no es sino una abstracción, un efecto, de la sociedad burguesa que habita. La emancipación humana, por el contrario, exige que los individuos conciban su libertad no como un derecho natural que antecede su existencia social, sino como el producto precisamente de su habitar al interior del ser social (Marx, 2004). Desplazando la noción de emancipación humana hacia lo desplegado en los Manuscritos, podemos decir que ésta exigiría de una "superación real" de la propiedad privada a través de una comprensión efectiva del ser genérico en tanto ser social e histórico ${ }^{8}$.

Para Jacques Rancière, la definición marxista de emancipación humana no deja de ser problemática. Esto por dos razones: en primer lugar, porque exige de una concepción teleológica de la historia. La emancipación humana, en tanto superación de la propiedad privada, sólo es posible gracias a la industria moderna, lo cual carga de necesidad el desarrollo histórico. En segundo lugar, Rancière postula que la emancipación humana, en la medida en que representa una superación real (hegeliana) del conflicto entre lo privado y lo público, constituye el fin del conflicto propiamente tal: así, mientras la sociedad civil se construye sobre el conflicto, la separación,

\footnotetext{
${ }^{8}$ Es importante hacer notar en este punto que en los Manuscritos Marx concibe el comunismo (la superación real de la propiedad privada) sólo como un paso en el progreso histórico y no como su finalidad: "El comunismo es la forma necesaria y el principio energético del inmediato futuro, pero el comunismo no es, en cuanto tal, la meta del desarrollo humano, la forma de la sociedad humana". Por el contrario, el socialismo sería "la auto-conciencia positiva del hombre" (Marx, 1968, p. 127).
} 
entre el individuo privado con sus intereses egoístas y el individuo público con sus responsabilidades ciudadanas, la emancipación real exige la superación efectiva de la separación misma. Para Rancière, por el contrario, el conflicto, la separación, la desigualdad, el desacuerdo, son todos lugares desde donde surge la política y por ende no es analogable la emancipación real con la desaparición del conflicto. Para comprender mejor tanto la crítica de Rancière al joven Marx como su propio concepto de emancipación, debemos comprender antes la diferencia que este autor instala entre los conceptos de "policía" y "política" (Rancière, 1996).

La policía es un término de clara herencia foucaultiana y que remite al ordenamiento de la sociedad bajo un concepto predeterminado, tecnocrático y racionalizado: "La policía es primeramente un orden de los cuerpos que define las divisiones entre los modos del hacer, los modos del ser y los modos del decir" (Rancière, 1996, p. 44). La política, en cambio, es una actividad antagónica a la de la policía: refiere precisamente a las prácticas que "rompen la configuración sensible donde se definen las partes y sus partes", generalmente provocada por la irrupción de aquellos "que no tienen parte" (Rancière, 1996, p. 45).

La violencia del orden policial reside en el hecho de que es un ordenamiento de lo sensible bajo una idea de totalidad. La política, por su parte, comienza allí donde ese orden policial es desarticulado, interrumpido. Esto ocurre a través del conflicto mismo, de la exigencia de igualdad de aquellos que fácticamente padecen la desigualdad ${ }^{9}$. A partir de esta definición de lo político como interrupción del orden policial podemos plantear que el conflicto mismo cumple una función fundacional para la política; por ello que la emancipación real, en tanto superación de la separación misma, no puede ser en sí política, sino meramente policial.

En el libro El espectador emancipado, Rancière (2009) desplaza sus postulados sobre la emancipación hacia el campo de las artes, en especial el teatro, no sólo como un mecanismo para pensar la relación entre arte y política, sino para reafirmar su propio concepto de emancipación. Si bien la "modernidad teatral" quiso acabar con la distancia entre el espectador y el actor, entre el artista y el público, entre el productor y el consumidor, y veía en ello una emancipación respecto del teatro burgués, Rancière vuelve

\footnotetext{
${ }^{9}$ El ejemplo utilizado por Rancière corresponde a la igualdad de las inteligencias. Si la escuela es precisamente un dispositivo policial que reproduce la desigualdad (ya que le hace creer a todos que su desigualdad es culpa de ellos mismos), la exigencia de una minoría de ser considerados de igual a igual sería una irrupción política a ese orden específico.
} 
a insistir en que la emancipación no acontece con la simple anulación de la distancia. Utilizando los ejemplos de Brecht y de Artaud, Rancière opone dos formas distintas de comprender la superación de la distancia entre el espectador y el espectáculo. En el primer caso, la superación es un acto cognitivo y reflexivo, que exige al espectador tomar conciencia de su lugar en la esfera de la producción y de las condiciones materiales de tal lugar; en el segundo caso, en cambio, la superación es una superación física, corporal, en la cual se borran los límites mismos entre el actor y el espectador.

Tal como en la crítica a la emancipación en Marx, Rancière se distancia de la concepción de que lo político en el arte resida simplemente en la superación de la distancia. Si la policía ordena y organiza lo sensible, el potencial político del arte consistiría antes que nada en su capacidad para cuestionar e interrumpir dicho orden. De este modo, la emancipación en el arte comienza precisamente allí donde comprendemos que el acto de contemplar es también una actividad en tanto que ordena y distribuye un contenido sensible. Y dado que toda contemplación es también actividad de ordenamiento sensible, entonces existiría en la relación entre espectador y espectáculo un tercer término, el mensaje mismo, sobre el cual ni espectador ni espectáculo tendrían control total (policial) y sobre cuya interpretación ambas partes incidirían (interrumpiendo políticamente el orden dado). La emancipación comienza entonces allí donde comprendemos que la relación espectador-actor no es una relación de sólo dos términos en la cual uno entrega un mensaje a otro: lo político del arte consiste precisamente en comprender que existe ese tercer factor entre el espectador y el espectáculo, el cual no puede ser "apropiado" por ninguna de las dos partes y gracias a lo cual puede resistir a la interpretación totalizante, a la lógica policial.

La crítica de Rancière nos permitiría otorgar un nuevo valor político a lo que he llamado "prácticas de emancipación digital" y que complementaría el análisis marxista ya realizado. Los tres ejemplos que dábamos de estas prácticas (software libre de fuente abierta, prácticas artísticas de postproducción, nuevos modos de producción musical) pueden ser leídos ahora no bajo la luz de la simple superación de la relación entre productor y usuario, sino como una serie de prácticas que desafían la oposición misma entre ambos. Estas tres nuevas lógicas de producción constituyen mecanismos de ordenamiento de lo sensible menos hegemónicos, y que interrumpen permanentemente la lógica policial. En el caso de la industria del software, por ejemplo, mientras las dos grandes corporaciones (Apple y Microsoft) ejercen una violencia simbólica que intenta hegemonizar un determinado "reparto de lo sensible", el software GNU ofrece el libre acceso 
no sólo a la "apropiación" del software, sino que ofrece además la posibilidad de modificarlo, y con ello desarticular dicho orden hegemónico. En el caso de la postproducción artística ocurre lo mismo: los productos de la industria cultural pueden ser "re-apropiados" y manipulados por software de edición y luego vueltos a distribuir vía Internet. En ambos casos se trata de dar cuenta de que existe en toda relación un tercer término que resiste a su apropiación policial.

Bajo la interpretación de Rancière, las prácticas de emancipación digital no significan una mera superación de la distancia, una mera abolición de la propiedad privada, sino que literalmente representan la crisis del derecho de autor, entendiendo por crisis no una destrucción ni una superación del dualismo, sino una libre circulación de ese tercero inapropiable que interrumpe permanentemente el orden policial que intenta hegemonizar nuestra relación sensible con lo real.

\section{REFERENCIAS}

Locke, John [1689] (1986). El segundo tratado sobre el gobierno civil. Buffalo: Prometeo.

Mandel, Ernest (1972). La formación del pensamiento económico de Marx. México: Siglo XXI.

Marx, Karl (1968). Manuscritos económico-filosóficos de 1844. México D.F.: Grijalbo.

(2004). Sobre la cuestión judía. Buenos Aires: Prometeo.

(2009). Elementos fundamentales para la crítica de la economía política. Volumen II. México D.F.: Siglo XXI.

Rancière, Jacques (1996). El desacuerdo. Política y filosofía. Buenos Aires: Nueva Visión. Verso.

(2009). El espectador emancipado. Trad. por Gregory Elliott. Londres:

Wu Ming (2005). “Copyright y maremoto", en Contra el copyright. México D.F.: Tumbona. 